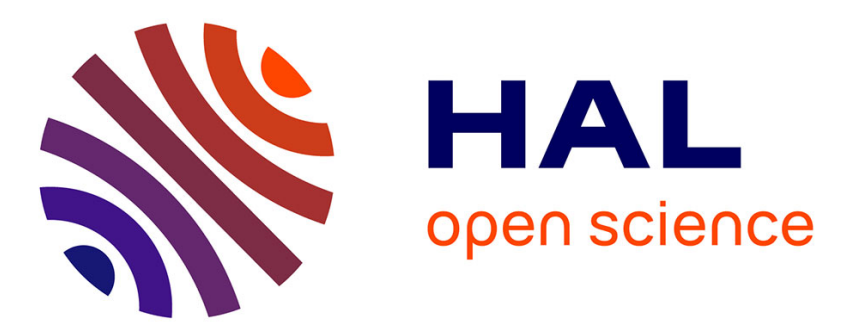

\title{
Metalliclike behavior of the exchange coupling in (001) $\mathrm{Fe} / \mathrm{MgO} / \mathrm{Fe}$ junctions
}

C. Bellouard, A. Duluard, Etienne Snoeck, Y. Lu, B. Negulescu, D. Lacour, C. Senet, S. Robert, N. Maloufi, S. Andrieu, et al.

\section{- To cite this version:}

C. Bellouard, A. Duluard, Etienne Snoeck, Y. Lu, B. Negulescu, et al.. Metalliclike behavior of the exchange coupling in (001) Fe/MgO/Fe junctions. Physical Review B: Condensed Matter and Materials Physics (1998-2015), 2017, 96 (13), pp.134416. 10.1103/PhysRevB.96.134416 . hal-01686936

\section{HAL Id: hal-01686936 \\ https://hal.univ-lorraine.fr/hal-01686936}

Submitted on 17 Jan 2018

HAL is a multi-disciplinary open access archive for the deposit and dissemination of scientific research documents, whether they are published or not. The documents may come from teaching and research institutions in France or abroad, or from public or private research centers.
L'archive ouverte pluridisciplinaire HAL, est destinée au dépôt et à la diffusion de documents scientifiques de niveau recherche, publiés ou non, émanant des établissements d'enseignement et de recherche français ou étrangers, des laboratoires publics ou privés. 


\title{
Metalliclike behavior of the exchange coupling in (001) $\mathrm{Fe} / \mathrm{MgO} / \mathrm{Fe}$ junctions
}

\author{
C. Bellouard, ${ }^{1, *}$ A. Duluard, ${ }^{1}$ E. Snoeck,,${ }^{2}$ Y. Lu,,${ }^{1}$ B. Negulescu, ${ }^{3}$ D. Lacour, ${ }^{1}$ C. Senet,,${ }^{1}$ S. Robert, ${ }^{1}$ N. Maloufi, ${ }^{4}$ S. Andrieu, ${ }^{1}$ \\ M. Hehn, ${ }^{1}$ and C. Tiusan ${ }^{5,6}$ \\ ${ }^{1}$ Institut Jean Lamour, UMR 7198, CNRS-Université de Lorraine, Boîte postale 230, 54506 Vandoeuvre-Les-Nancy, France \\ ${ }^{2}$ CEMES CNRS, 29 Rue Jeanne Marvig, F-31055 Toulouse, France \\ ${ }^{3}$ Groupe de Recherche en Matériaux, Microélectronique, Acoustique et Nanotechnologies, UFR Sciences et techniques, Université de Tours, \\ Parc de Grandmont, Batiment E, 37200, TOURS, France \\ ${ }^{4}$ Université de Lorraine, Laboratoire d'Etude des Microstructures et de Mécanique des Matériaux LEM3, \\ UMR 7239, F-57045 Metz cedex 1, France \\ ${ }^{5}$ Technical University of Cluj-Napoca, Center of Superconductivity, Spintronics and Surface Science, Str. Memorandumului 28, \\ RO-400114 Cluj-Napoca, Romania \\ ${ }^{6}$ Centre National de la Recherche Scientifique, DGDR, DR06, France
}

(Received 29 July 2017; revised manuscript received 19 September 2017; published 12 October 2017)

\begin{abstract}
Exchange magnetic coupling between Fe electrodes through a thin $\mathrm{MgO}$ interlayer in epitaxial junctions has been investigated as a function of temperature, $\mathrm{MgO}$ thickness, and interface quality. Depending on the $\mathrm{MgO}$ thickness, which has been varied from 1.5 to 4 monolayers, two opposite temperature dependences are clearly disentangled. For a thin $\mathrm{MgO}$ spacer, the main component decreases with temperature following a metalliclike behavior. On the contrary, for the thickest $\mathrm{MgO}$ layers, the main component increases with temperature, following an Arrhenius law. Moreover, the insertion of a monoatomic roughness at the bottom $\mathrm{MgO}$ interface, induced by the addition of a fraction of a Fe monolayer, exacerbates the metallic features as an oscillatory behavior from antiferromagnetic to ferromagnetic is observed. These results allow questioning the simple tunneling mechanism usually invoked for $\mathrm{MgO}$ coupling, and suggest a crossover behavior of the thin $\mathrm{MgO}$ spacer from metallic to insulating with a progressive opening of the gap.
\end{abstract}

DOI: 10.1103/PhysRevB.96.134416

\section{INTRODUCTION}

Interlayer exchange coupling (IEC) across a nonmagnetic spacer is a useful tool to control magnetic alignment of ferromagnetic electrodes in spintronic devices. Concerning the metallic spacer, systematic results have been established and its behavior, for instance, as a function of layer thickness, is well understood [1,2]. On the contrary, for a semiconducting or insulating spacer [3-8], magnetic properties seem to depend deeply on growth conditions and then on structural specifications. Among them, the $\mathrm{MgO}$ spacer [9-24] appears unique in presenting a large variety of experimental results. They concern either the $\mathrm{MgO}$ thickness range and the coupling sign and strength, or the junction magnetic geometry (planar or perpendicular magnetization).

The first results were obtained with epitaxial planar junctions grown by evaporation, with a very thin $\mathrm{MgO}$ spacer, and concern mainly an antiferromagnetic (AF) coupling $[9,10,16,20]$. The maximum AF coupling strength ranges from $0.04 \mathrm{erg} / \mathrm{cm}^{2}$ [10], for a $\mathrm{MgO}$ thickness close to 3 monolayers, to $1.2 \mathrm{erg} / \mathrm{cm}^{2}$ [20,25], for a crossover towards the ferromagnetic pinholes regime occurring at a thickness as low as 1 monolayer [20]. This crossover is obviously governed by the topography of the interface which can be strongly modified by a $\mathrm{MgO}$ buffer deposited on a $\mathrm{MgO}$ substrate [20]. Moreover, the presence of oxygen through iron oxide electrodes $[11,25]$ or interface oxidation $[12,18]$ influences bilinear and/or biquadratic coupling. We point out that a weaker ferromagnetic coupling $\left(<0.03 \mathrm{erg} / \mathrm{cm}^{2}\right)$ has also been

*christine.bellouard@univ-lorraine.fr reported for $\mathrm{MgO}$ thicknesses close to $1 \mathrm{~nm}[9,10,24]$. In the case of sputtered junctions, a ferromagnetic coupling has been observed in the planar magnetic configuration with an exponential $\mathrm{MgO}$ thickness dependence [13,17], and has therefore been attributed to a cooperative electronic effect. Nevertheless, an antiferromagnetic coupling $\left(<0.1 \mathrm{erg} / \mathrm{cm}^{2}\right)$ has been observed recently with a sputtered planar multilayer with a quite large $\mathrm{MgO}$ thickness (1.6 nm) [23].

Such antiferromagnetic coupling across a $\mathrm{MgO}$ spacer has also been observed with perpendicular magnetic junctions grown by sputtering $[14,15,19,21,22]$. Nevertheless, its features are then much different than those of planar epitaxial junctions. The AF coupling is indeed observed for quite large thicknesses $(\geqslant 1.2 \mathrm{~nm})$, with amplitude about two orders of magnitude lower than the values of the epitaxial junctions. Moreover, a surprising oscillatory behavior was observed as a function of the magnetic layer thickness [14,19,21].

As a consequence of this variety of experimental results, different theoretical approaches have been proposed. The free-electron model relevant for a metallic spacer [1] can be extended to an insulating barrier $[2,26]$. This approach, firstly used to interpret the $\mathrm{Fe} / \mathrm{MgO} \mathrm{AF}$ coupling because of its strong thickness dependence [9], is now dismissed [10] as this model is not valid for such a thin barrier. It has been more satisfactorily applied in the case of planar or perpendicular junctions with a thicker barrier [17]. Nevertheless, the temperature dependence predicted by Bruno [2,27] in the case of an insulating barrier has never been clearly observed. The experimental results are rather close to what is found across a semiconducting spacer $[15,21]$. On the other hand, for low $\mathrm{MgO}$ thicknesses, as in planar-evaporated $\mathrm{Fe} / \mathrm{MgO}$ 
junctions, Zhuralev et al. [28,29] found that the coupling should be ferromagnetic, and the antiferromagnetic coupling could be due to a resonant tunneling mechanism, mediated, for instance, by defects in the barrier such as oxygen vacancies. Nevertheless, $a b$ initio calculations results depend on the supercell description and atomic distances $[29,30]$.

Beyond these theoretical approaches, intrinsic mechanismor defects-mediated processes across insulating barriers would lead to different temperature behavior. Surprisingly, few temperature studies have been carried out $[8,12,15,22]$. The present experimental work is then devoted to a systematic temperature study of the bilinear coupling performed with epitaxial $\mathrm{Fe} / \mathrm{MgO}$ planar junctions grown in optimal conditions. Two opposite temperature dependences have been clearly identified. Moreover, a controlled structural disorder has been introduced by inserting a Fe layer, submonolayer or a few monolayers thick, on the Fe buffer. For a thickness lower than 1 monolayer (ML), this results in decorating the buffer with atomic thick islands. Unexpectedly, it is found that this atomic scaled controlled disorder can amplify the antiferromagnetic coupling, or lead to the appearance of a ferromagnetic coupling, depending on the $\mathrm{MgO}$ thickness.

In the present work, two junction architectures have been studied. The first magnetic stack consists of thick/thin $\mathrm{Fe}$ layers: $\mathrm{Fe}(50 \mathrm{~nm}) / \mathrm{MgO}(x \mathrm{ML}) / \mathrm{Fe}(5 \mathrm{~nm})$. In that case, only the antiferromagnetic coupling can be investigated with the reversing of the thinnest layer in a positive field. Nevertheless, it allows precise measurements of the coupling with high reversing field, particularly as a function of temperature, and the study of a wedge sample with Kerr magnetometry. The second type consists of a soft/hard magnetic stack: Fe $(50 \mathrm{~nm}) / \mathrm{MgO}(x \mathrm{ML}) / \mathrm{Fe}(5 \mathrm{~nm}) / \mathrm{Co}(100 \mathrm{~nm})$. In that case, both ferromagnetic and antiferromagnetic coupling can be investigated, with the reversing of the Fe bottom layer.

The paper is organized as follows: After some experimental details, structural investigation using high-resolution transmission microscopy is presented. The temperature study of the antiferromagnetic bilinear coupling measured with the thick/thin stack and flat interface is then surveyed. Thereafter, the effect of the insertion of artificial roughness upon the Fe buffer is examined. The temperature dependence is then investigated with a soft/hard stack. Finally, a discussion of the complete results supported by $a b$ initio calculations is proposed.

\section{EXPERIMENT}

\section{A. Sample preparation}

$\mathrm{The} \mathrm{Fe} / \mathrm{MgO} / \mathrm{Fe}$ junctions are grown by molecular beam epitaxy (MBE). Previous to deposition, the (001)MgO substrate was outgassed at $700{ }^{\circ} \mathrm{C}$ for $60 \mathrm{~min}$, and covered by a 10-nm MgO trapping layer, which prevents carbon diffusion from the substrate to the stack during buffer annealing [31]. All layers have been grown by evaporation from an electron gun, with a quartz oscillating thickness controller. For both types of junctions described above, the thicknesses of the magnetic layers have been checked ex situ by x-ray reflectometry. The bottom $\mathrm{Fe}$ layer $(50 \mathrm{~nm})$ is annealed at $580^{\circ} \mathrm{C}$ for $20 \mathrm{~min}$ to flatten its surface. We point out that the $\mathrm{Fe}$ bottom layer is identical for both stack architectures to warrant the same growth conditions of the $\mathrm{MgO}$ spacer. An in situ moving shutter allows us to grow $\mathrm{Fe}$ or $\mathrm{MgO}$ wedges, or to part the substrate in different zones. The thickness of the $\mathrm{MgO}$ barrier has been firstly calibrated with oscillations of reflection of high-energy electron diffraction (RHEED) intensity. This thickness is afterward controlled with a quartz oscillating sensor and is reproducible from one sample to another (grown on different substrates) with an absolute error estimated to about $0.2 \mathrm{ML}$. The roughness of the $\mathrm{MgO}$ bottom interface has been modulated with the insertion of a $0-3$ Fe ML. Its thickness is calibrated in situ for each sample with RHEED intensity oscillations. After this calibration, the sample is again annealed at $580{ }^{\circ} \mathrm{C}$. The $\mathrm{Fe}$ islands are then deposited at $100^{\circ} \mathrm{C}$. Thereafter, no subsequent annealing is performed during the junction growth.

The stacks are protected by a 20 -nm-thick Au layer for magnetic and structural measurements, and with a 3-nm-thick Au layer for Kerr magnetometry.

\section{B. Sample characterization}

High-resolution transmission electron microscopy: The structures of the $\mathrm{MgO}$ and $\mathrm{Fe}$ layers and $\mathrm{Fe} / \mathrm{MgO}$ interfaces were investigated by transmission electron microscopy (TEM) in high-resolution mode (HRTEM) using a Tecnai F20 (FEI) fitted with an objective lens corrected for spherical aberration (CEOS). The cross-sectional specimens for TEM studies were cut along $\mathrm{MgO}$ (100) planes. They were glued face to face and thinned using tripod polishing and ion milling at low angle and low voltage to achieve the electron transparency.

Magnetic measurements were performed with a SQUIDVSM (Quantum Design) magnetometer, in the field range $\pm 1.5 \mathrm{kOe}$, with a standard stick in the 5-300 $\mathrm{K}$ temperature range, and with an oven stick for temperatures above $300 \mathrm{~K}$.

Kerr magnetometry has been performed with a commercial Kerr microscope from Evico Magnetics at room temperature, in the $\pm 1 \mathrm{kOe}$ field range. The data have been recorded in the so-called longitudinal geometry in which the magnetic field is applied along the intersection of the sample plane with the incident plane of the light.

All magnetic measurements (Kerr, SQUID) have been performed with the magnetic field applied, in the sample plane, along an easy axis [100] of iron.

\section{RESULTS AND DISCUSSION}

\section{A. High-resolution transmission electron microscopy}

Typical HRTEM images are shown in Figs. 1(a) and 1(b) for a junction with a flat $\mathrm{Fe} / \mathrm{MgO}$ bottom interface and in Figs. 1(c) and 1(d) for a junction with $\mathrm{Fe}$ islands, corresponding to the insertion of a $0.5 \mathrm{Fe}$ ML. The HRTEM results confirm the expected epitaxial relationship $\mathrm{Fe}(001)[110] / / \mathrm{MgO}(001)[100]$ with the bcc cubic structure of $\mathrm{Fe}$ and fcc of $\mathrm{MgO}$ rotated by $45^{\circ}$. With or without $\mathrm{Fe}$ islands, no clear pinhole is observed. The $\mathrm{MgO}$ barrier is always continuous with zones of wellordered atomic alignment in the direction perpendicular to the interface as in Figs. 1(a) and 1(c). With or without islands, the 3-ML-thick MgO layer is strained by the underlying buffer. Reflection of high-energy electron diffraction (RHEED) has 

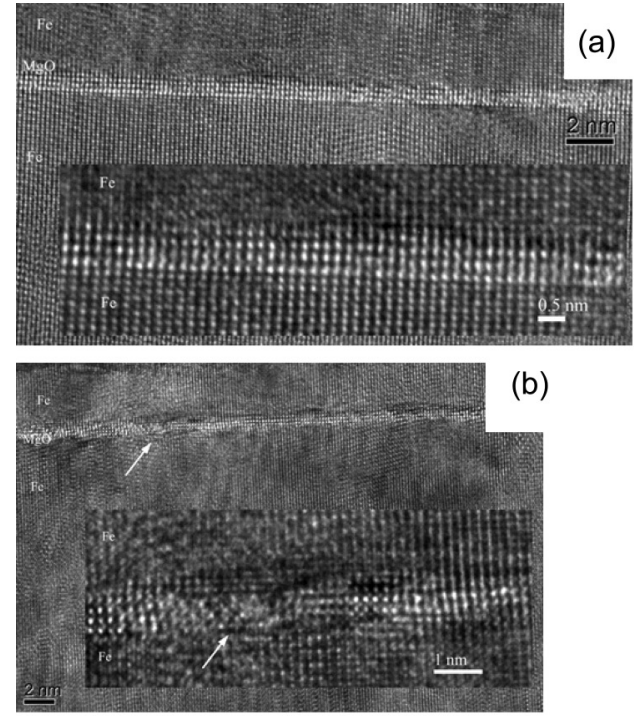
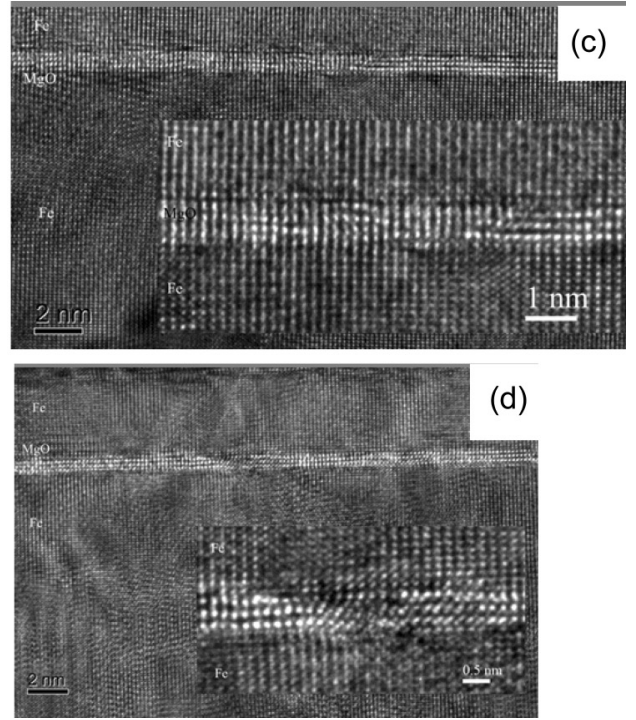

FIG. 1. High-resolution transmission microscopy of $\mathrm{Fe}(50 \mathrm{~nm}) / \mathrm{Fe}(x) / \mathrm{MgO}(3.1 \mathrm{ML}) / \mathrm{Fe}(5 \mathrm{~nm})$ where $x=0 \mathrm{ML}$ or $0.5 \mathrm{ML}$. (a,b) correspond to a flat $\mathrm{Fe} / \mathrm{MgO}$ interface and (c,d) to an interface with $\mathrm{Fe}$ islands.

shown that the plastic relaxation occurs in a similar way in both cases for a larger $\mathrm{MgO}$ thickness [32]. Moreover, from this study [32], an average island size of $6 \mathrm{~nm}$ could be estimated.

Some stacking defects (atomic displacements) are observed within the $\mathrm{MgO}$ layer and at the $\mathrm{Fe} / \mathrm{MgO}$ interfaces as shown in Figs. 1(b) and 1(d). Nevertheless, and even considering difficult performing statistic measurements in TEM experiments, we do not evidence a higher density of such defects in junctions with $\mathrm{Fe}$ islands. These observations corroborate the growth study performed with RHEED [32]. Even with thin $\mathrm{MgO}$ layers (3 ML), the thickness of the barrier remains homogeneous when deposited on a Fe surface decorated with atomic Fe islands.

\section{B. Temperature study}

Figure 2(a) presents a typical magnetization loop of a Fe $(50 \mathrm{~nm}) / \mathrm{MgO}(3 \mathrm{ML}) / \mathrm{Fe}(5 \mathrm{~nm})$ junction. After saturation under $2 \mathrm{kOe}$, the thin layer reverses spontaneously in a positive field with a two-step process, as a result of competition between Zeeman and coupling energy. The minor loop of the thinnest layer [inset of Fig. 2(a)] is indeed characterized by the saturation field $H_{s}$ where the junction leaves its parallel state, and the plateau field $H_{p}$ below which the junction is antiparallel. In between these fields, an in-plane perpendicular configuration of the layer is stabilized. This intermediate state has been attributed to the fourfold anisotropy of iron [33]. It could also arise from the contribution of a biquadratic coupling [12].

As discussed in Appendix A, in the case of two magnetic layers in thin/thick or soft/hard stacks, the bilinear coupling can be deduced from $H_{S}$ and $H_{P}$ as

$$
J(T)=-M_{S}(T) t \frac{H_{S}+H_{P}}{2}(T),
$$

where $M_{S}(T)$ is the temperature-dependent magnetization of the reversing layer as determined on the magnetization loops, and $t$ its thickness. The decrease of $M_{S}(T)$ of the 5-nm-thick


FIG. 2. (a) Magnetization loop of a $\mathrm{Fe} \quad(54.0 \mathrm{~nm}) / \mathrm{MgO}$ (3 ML)/Fe $(4.3 \mathrm{~nm})$ junction measured at $300 \mathrm{~K}$. Inset: minor loop of the thinnest layer where the switching fields are pointed out with dotted lines; an arrow points to the saturation field $H_{s}$ and plateau field $H_{P}$; they correspond to the average value of the switching fields from or towards saturation (respectively, plateau) magnetization. (b) Magnetization loop of a Fe $(50 \mathrm{~nm}) / \mathrm{MgO}(2.5 \mathrm{ML}) / \mathrm{Fe}(5 \mathrm{~nm}) / \mathrm{Co}$ $(100 \mathrm{~nm})$ measured at $5 \mathrm{~K}$. 




FIG. 3. Temperature dependence of the bilinear antiferromagnetic coupling $-J$ of three $\mathrm{Fe}(50 \mathrm{~nm}) / \mathrm{MgO}(x \mathrm{~nm}) / \mathrm{Fe}(5 \mathrm{~nm})$ junctions with (a) $x=3.0 \mathrm{ML}$; (b) $3.25 \mathrm{ML}$; (c) $3.5 \mathrm{ML}$. The straight lines are fits with the model described in the text.

Fe layer, as deduced from magnetization measurements, is lower than $4 \%$.

The biquadratic coupling is then a function of the difference $H_{S}-H_{P}$ (Appendix A).

Figure 2(b) shows a magnetization loop of a $\mathrm{Fe}$ $(50 \mathrm{~nm}) / \mathrm{MgO}(2.5 \mathrm{ML}) / \mathrm{Fe}(5 \mathrm{~nm}) / \mathrm{Co}(100 \mathrm{~nm})$ junction. In that case, the bottom Fe layer firstly reverses with decreasing field after saturation. The coupling is now deduced using the $50-\mathrm{nm}$ Fe layer, whereas it was deduced from the reversing of a 5-nm-thin layer with the thin/thick stack. As a matter of fact, for similar coupling strengths, the reversing fields $H_{S}$ and $H_{P}$ are then much lower [see Eq. (1)].

The present study is devoted to the bilinear coupling. The results concerning the biquadratic one are therefore presented in Appendix B.

As the main part of the paper is devoted to the antiferromagnetic coupling, $J<0$, only the absolute value or amplitude $|J|=-J$ will be considered in the following, except at the end of the paper, in Fig. 7.

The temperature dependence of the bilinear coupling is plotted in Fig. 3 for three junctions with different $\mathrm{MgO}$ thicknesses. A continuous decrease of the coupling constants with increasing temperature is observed for a gap of $3 \mathrm{ML}$ [Fig. 3(a)] whereas a nonmonotonous dependence appears for larger thicknesses [Figs. 3(b) and 3(c)]. The decrease of the coupling with increasing temperature has been confirmed by high-temperature measurements presented in Appendix C. It corresponds to the usual dependence of the coupling across a metallic layer [2]. To quantify this dependence, we have used a phenomenological model deduced from the theory of Bruno, which satisfactorily fits the temperature dependence [Fig. 3(a)] with only two parameters $J^{\mathrm{EXC}}(0)$ and $T^{0}$ :

$$
J^{\mathrm{EXC}}(T)=J^{\mathrm{EXC}}(0) T^{0} \int_{0}^{1 / T^{0}} \frac{\alpha T}{\sinh (\alpha T)} d \alpha .
$$

$J^{\mathrm{EXC}}(0)$ stands for the so-called "exchange coupling" (EXC) intensity at $0 \mathrm{~K}$, and $T^{0}$ characterizes its temperature dependence.

In the free-electron model developed by Bruno [2], $\alpha$ is related to electron parameters $m$ and $k_{F}$, and to the spacer width $D$ as

$$
\alpha=\frac{\hbar^{2} k_{F}}{2 \pi k_{B} D m} .
$$

The temperature dependence of coupling in the model of Bruno solely comes from the Fermi-Dirac distribution width. It does not take into account any thermal excitations of magnetization.

The temperature increasing contribution, clearly appearing for thicker $\mathrm{MgO}$ layers, reminds us of the temperature dependence observed with an amorphous semiconducting spacer such as Si [34], Ge [35], or ZnSe [4]. To take into account this contribution, a simple Arrhenius law can be added, according to the following equation:

$$
\begin{aligned}
J(T)= & {\left[J^{\mathrm{EXC}}(0)+J^{\text {th }} \exp \left(-\frac{E}{k_{B} T}\right)\right] T^{0} } \\
& \times \int_{0}^{1 / T^{0}} \frac{\alpha T}{\sinh (\alpha T)} d \alpha,
\end{aligned}
$$

where $J^{\text {th }}$ is the thermally activated coupling contribution, and $E$ the energy barrier.

More work needs to be done in devising a theoretical description of the coupling regime where the interlayer spacer is neither metal nor insulator. Our choice for introducing this Arrhenius term as a prefactor in Eq. (3) has the following physical justification. The shape of the thermal variation of $J$ for the samples with thicker $\mathrm{MgO}$ barrier, where we observe an increase of coupling with increasing the temperature, cannot be fitted using a standard tunneling coupling mechanism [Eq. (2) adapted for tunneling regime where the temperature dependence would be in this case $\alpha T / \sin (\alpha T)$ ] [2]. On the other hand, this temperature variation could be phenomenologically well described by an Arrhenius thermal activation law. This term, introduced as a prefactor, could describe the intermediate regime where the spacer is a small-gap semiconductor and electrons could be therefore thermally excited in its conduction band. Therefore, phenomenologically, one can adapt Eq. (3) deduced from the model of Bruno, which described the coupling across a metallic spacer, to coupling across a low-gap semiconductor. The Arrhenius factor would describe the thermal dependence of the carrier density participating in the spin currents and implicitly giving rise to the spin torque, the exchange coupling being the zero bias (equilibrium) component of the field like-spin torque. 




FIG. 4. $\mathrm{MgO}$ thickness dependence of the parameters deduced from the fit of the temperature dependence of the coupling constant $j(T)$ for a $\mathrm{Fe}(50 \mathrm{~nm}) / \mathrm{MgO}(x \mathrm{~nm}) / \mathrm{Fe}(5 \mathrm{~nm})$ junction. $(\mathrm{a}, \mathrm{b})$ Exchange coupling; (c,d) thermally activated coupling. The dotted lines are guides for the eyes. The error bars correspond to a confidence level of $95 \%$.

The experimental points in Figs. 3(b) and 3(c) are satisfactorily fitted by Eq. (3) (see also Fig. 13). The fitted parameters are plotted in Fig. 4: $J^{\mathrm{EXC}}(0), T^{0}$ for EXC coupling, and $J^{\text {th }}$, $E$ referring to the "thermally activated coupling."

Concerning the temperature dependence of the exchange term, the values of $T^{0}$ [Fig. 4(a)] are significantly larger than what is observed for the metallic spacer in the case of Ruderman-Kittel-Kasuya-Yoshida (RKKY) coupling (90$100 \mathrm{~K})$ [36-39]. Moreover, it increases slightly with the $\mathrm{MgO}$ thickness.

In the present case, it corresponds to the lower value of a distribution ranging from $T^{0}$ to infinity, according to Eq. (3). This means that the temperature dependence of the EXC coupling through $\mathrm{MgO}$ is flatter than in the case of huge metallic coupling. It behaves as an intermediate case between a true metallic and an insulating spacer for which a flat dependence with temperature is rather expected [2].
These results strengthen a posteriori the proposed analysis. The corresponding coupling intensity $\left(\left|J^{\mathrm{EXC}}\right|=-J^{\mathrm{EXC}}\right)$ decreases towards zero with the covering of the fourth plane. The thermally activated term appears for a $\mathrm{MgO}$ layer thickness higher than three planes. Opposite to the EXC term, the "thermal" coupling intensities are almost independent of the $\mathrm{MgO}$ thickness, from its onset, for $t_{\mathrm{MgO}}$ higher than $3.2 \mathrm{ML}$, and until $4 \mathrm{ML}$. Within the error bars, the activation energy appears also independent of the $\mathrm{MgO}$ thickness. The thermal component exhibits then a $\mathrm{MgO}$ thickness dependence far from the one of the exchange term, and cannot therefore be understood as a simple thermal contribution to this term. The physical origin of this thermal activated term remains puzzling; one cannot rule out a contribution of interfaces in this mechanism.

\section{Atomic roughness effect}

First of all, the influence of the quality of the Fe buffer/$\mathrm{MgO}$ spacer interface has been investigated by inserting, between the annealed buffer and the $\mathrm{MgO}$ layer, a Fe wedge ranging from 0 to $3 \mathrm{ML}$. The evolution of the coupling constant along the wedge has been measured at room temperature by Kerr magnetometry for three $\mathrm{MgO}$ thicknesses. In that case, the antiferromagnetic coupling constants are deduced from the reversing field of the thin layer of the stack: $\mathrm{Fe}(50 \mathrm{~nm}) / \mathrm{MgO}$ $(t) / \mathrm{Fe}(5 \mathrm{~nm})$.

The results are reported in Fig. 5. It exhibits nice oscillations of the coupling constant with the $\mathrm{Fe}$ covering rate with a

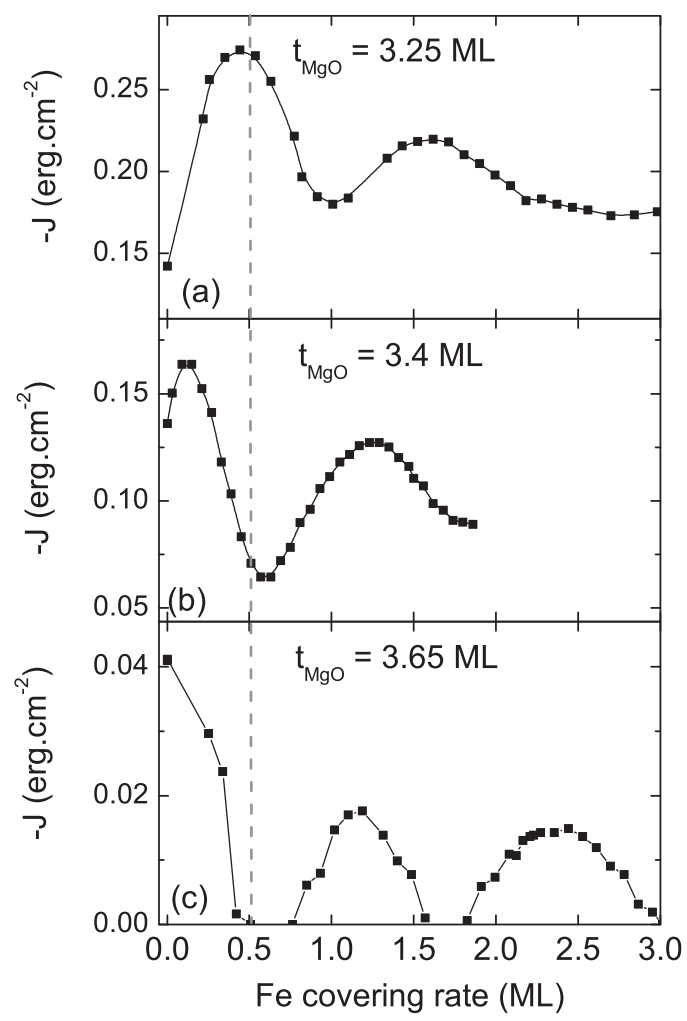

FIG. 5. Antiferromagnetic bilinear coupling constant measured at room temperature by Kerr magnetometry as a function of the $\mathrm{Fe}$ covering rate of the Fe buffer for three $\mathrm{MgO}$ spacer thicknesses: (a) 3.25 ML; (b) $3.4 \mathrm{ML}$; (c) $3.65 \mathrm{ML}$. The stack of the sample is Fe $(50 \mathrm{~nm}) / \mathrm{MgO}(t \mathrm{ML}) / \mathrm{Fe}(5 \mathrm{~nm})$. 
period close to $1 \mathrm{ML}$. A global decrease of the maximum is observed with an increasing $\mathrm{Fe}$ covering rate towards 3 ML [Fig. 5(a)]. This feature is expected with increasing topographical interface disorder. This introduction of several Fe monolayers allows indeed to model some interdiffused region at the interface as in sputtered junctions [40] where the magnetic coupling is lower [14,23].

Interestingly, for a spacer of 3.25 ML [Fig. 5(a)], the antiferromagnetic coupling is enhanced with the introduction of $0.5 \mathrm{ML}$ Fe. This amplification of the coupling with the introduction of some disorder is counterintuitive. For a larger $\mathrm{MgO}$ thickness [3.65 ML, Fig. 5(c)], the antiferromagnetic coupling appears quenched by the introduction of an atomic roughness (at $0.5 \mathrm{Fe} \mathrm{ML}$ covering rate or $50 \%$ covering area), resulting in an oscillation in opposite phase with Fig. 5(a). As a consequence, a phase displacement of the oscillations is observed with increasing $\mathrm{MgO}$ thickness as observed from Figs. 5(a)-5(c).

This complex behavior observed at $300 \mathrm{~K}$ sketches the inconsistency of experimental results and the huge dependence of the $\mathrm{MgO}$ IEC upon sample preparation conditions. In order to disentangle the different components of the coupling, a temperature study has been performed as a function of $\mathrm{MgO}$ thickness with atomic rough interface $(0.5 \mathrm{Fe} \mathrm{ML})$. It has been performed with a soft/hard stack to investigate both ferromagnetic and antiferromagnetic coupling.

Figure 6(a) presents the temperature dependence of the coupling with a 3.4-ML $\mathrm{MgO}$ spacer and a flat interface. As compared with the results plotted in Fig. 3, the qualitative behavior is similar. It is nicely fitted with Eq. (3), providing parameters characterizing the temperature dependence of the EXC $\left(T^{0}=140 \mathrm{~K}\right)$ and thermal coupling $\left(E / k_{B}=73 \mathrm{~K}\right)$ in agreement with the previous results. On the contrary, the coupling constants $J^{\mathrm{EXC}}\left(0.21 \mathrm{erg} / \mathrm{cm}^{2}\right)$ and $J^{\text {th }}\left(0.13 \mathrm{erg} / \mathrm{cm}^{2}\right)$ are larger. One could think about an effect of the magnetic layer thickness on the coupling strength, as the magnetic layers are both thick in the soft/hard stack. Nevertheless, the dependence of the coupling with the magnetic layer thickness is not investigated in the present work. Figures $6(\mathrm{~b})$ and 6(c) present the temperature dependence of junctions with interfacial Fe islands. They are still very well fitted with Eq. (3) with parameters $T^{0}$ (see Fig. 6 caption) consistent with Fig. 4. The energy barriers $E$ for 2.6 and $3.9 \mathrm{MgO} \mathrm{ML}$ agree with the mean value of the flat interface [Fig. 4(d)].

As a conclusion, the parameters characterizing the temperature dependence, $T^{0}$ and $E$, appear then as intrinsic to the $\mathrm{MgO}$ layer and its thickness, whereas amplitudes of both components of the coupling (EXC and thermal) are strongly dependent on the interface quality and magnetic layers' thicknesses.

The $\mathrm{MgO}$ thickness dependence of the coupling in the soft/hard stack is plotted in Fig. 7. As was illustrated in Fig. 2(b), the coupling constant is deduced from the reversing field of the Fe buffer layer.

The four curves of Fig. 7 correspond to flat interface $(0$ Fe ML) or rough one (with $0.5 \mathrm{Fe} \mathrm{ML}$ ), each measured at 5 and $300 \mathrm{~K}$. They all exhibit qualitatively a $\mathrm{MgO}$ thickness dependence close to previous papers $[9,10]$. A maximum of the antiferromagnetic coupling strength $|J|$ is found around 2 $\mathrm{MgO} \mathrm{ML}$ as a crossover between antiferromagnetic coupling

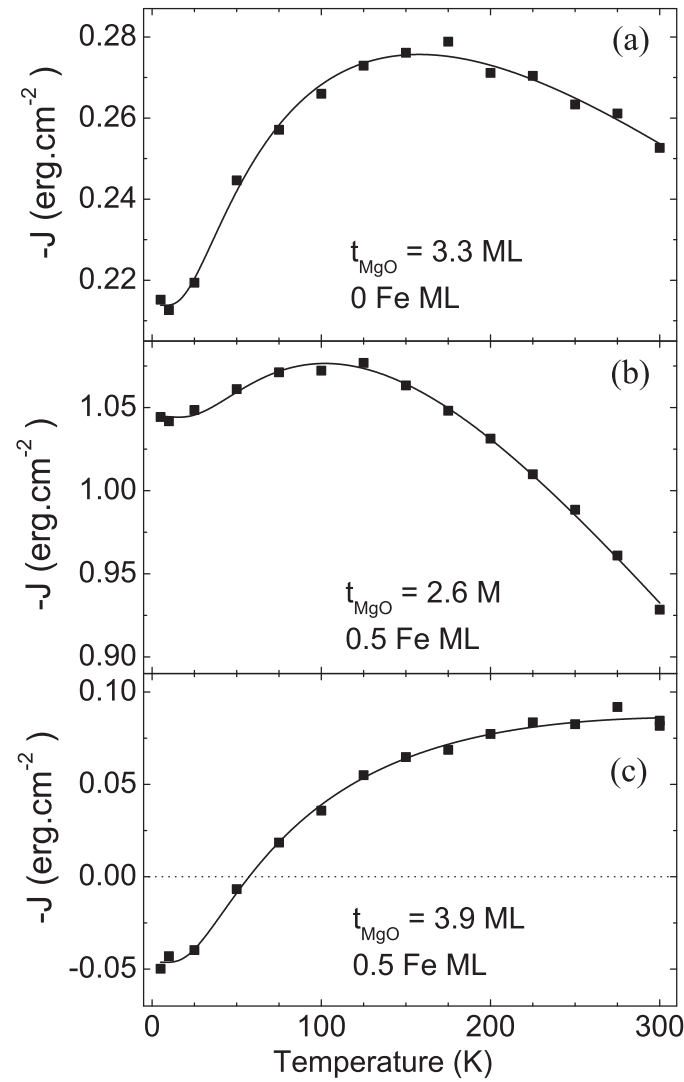

FIG. 6. Temperature dependence of the coupling in soft/hard $\mathrm{MgO}$ junctions with different $\mathrm{MgO}$ thicknesses. (a) Flat interface; (b,c) atomic rough interface. The lines are fits using Eq. (3). The fitted parameters are (a) $T^{0}=140 \mathrm{~K},-J^{\mathrm{EXC}}(0)=0.21 \mathrm{erg} / \mathrm{cm}^{2}$, $-J^{\text {th }}=0.13 \mathrm{erg} / \mathrm{cm}^{2}, E=73 \mathrm{~K}$; (b) $T^{0}=134 \mathrm{~K},-J^{\mathrm{EXC}}(0)=$ $1.04 \mathrm{erg} / \mathrm{cm}^{2},-J^{\text {th }}=0.185 \mathrm{erg} / \mathrm{cm}^{2}, E=105 \pm 20 \mathrm{~K}$; (c) $T^{0}=$ $150 \pm 30 \mathrm{~K},-J^{\mathrm{EXC}}(0)=-0.046 \mathrm{erg} / \mathrm{cm}^{2},-J^{\text {th }}=0.20 \mathrm{erg} / \mathrm{cm}^{2}$, $E=83 \mathrm{~K}$.

coming from the $\mathrm{MgO}$ spacer and ferromagnetic coupling coming from pinholes (ferromagnetic bridges across the $\mathrm{MgO}$ layer). The antiferromagnetic coupling amplitude $|J|$ then strongly decreases for a $\mathrm{MgO}$ thickness above 2.5 ML. Moreover, two specific features can be pointed out. The first one concerns the exact position of the maximum which is shifted from about $0.5 \mathrm{ML}$ towards larger MgO thickness in the case of a rough interface. Despite no discrepancies having been observed in the HRTEM experiment, it seems reasonable to attribute this shift to a stronger ferromagnetic coupling induced by pinholes in the case of a disordered interface. The second point concerns the amplitude of the antiferromagnetic coupling which is larger than previously reported $[9,20]$.

We now focus on the temperature dependence. As reported above, for low $\mathrm{MgO}$ thicknesses (below 2.5 ML), the amplitude $|J|$ of the antiferromagnetic coupling decreases between 5 and $300 \mathrm{~K}$, for both interfaces. It has been related to a metalliclike behavior. On the contrary, for larger thicknesses, when the temperature increases from 5 to $300 \mathrm{~K}$, an antiferromagnetic component has to be added; this is due to the contribution of the thermal component as discussed above. One can notice that for $\mathrm{MgO}$ thicknesses above 3.7 ML, this component is enhanced with the presence of $\mathrm{Fe}$ atomic islands. Moreover, focusing 


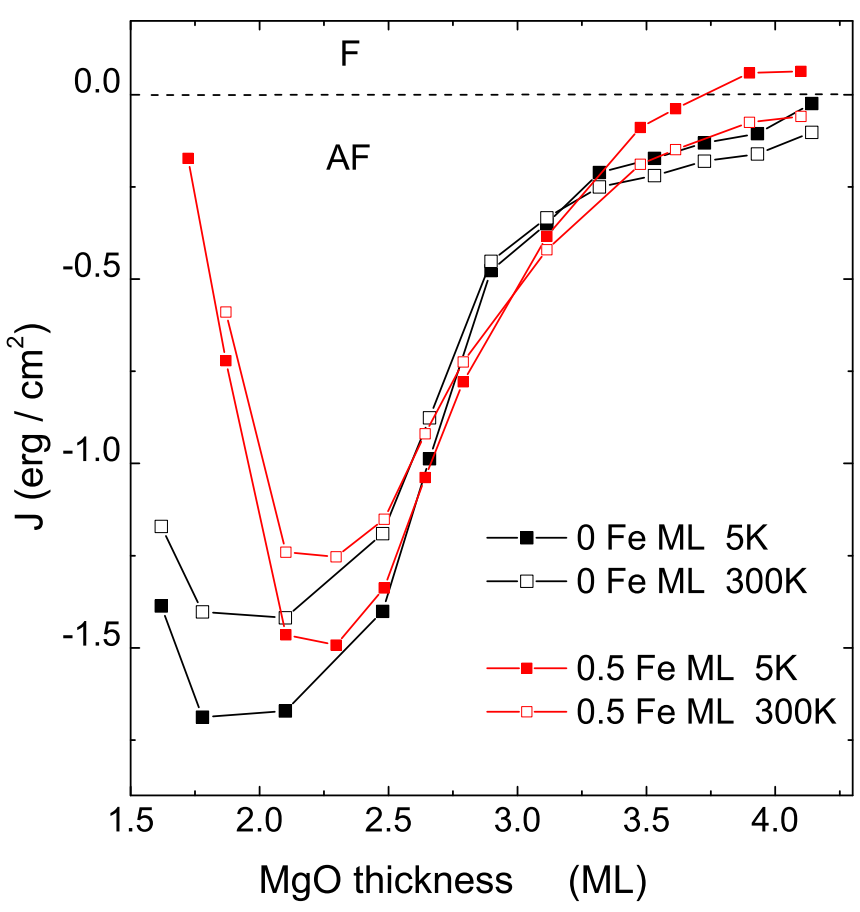

FIG. 7. Mean interlayer exchange coupling measured at 5 and $300 \mathrm{~K}$ as a function of $\mathrm{MgO}$ thickness wedge in the case of a $(\mathrm{Fe}$ $50 \mathrm{~nm} / \mathrm{MgO} x / \mathrm{Fe} 5 \mathrm{~nm} / \mathrm{Co} 100 \mathrm{~nm}$ ) stack with flat (0 Fe ML) or atomic rough $(0.5 \mathrm{Fe} \mathrm{ML})$ interface.

on the room-temperature behavior, a crossing between flat and rough interface coupling is observed around 3.5-3.7 $\mathrm{MgO} \mathrm{ML}$, the $\mathrm{MgO}$ thickness dependence being steeper for the atomic rough interface. This statement agrees with the behavior of the oscillations described by Fig. 5 .

Finally, we focus on the low-temperature dependence ( $5 \mathrm{~K}$ ) which corresponds exclusively to the EXC component. Above 2.7 $\mathrm{MgO} \mathrm{ML}$, where the influence of pinholes is negligible for both interfaces, the coupling in the presence of atomic roughness presents a much steeper variation than the reference one, and even becomes ferromagnetic around $4 \mathrm{MgO}$ ML, with a value as large as $0.05 \mathrm{erg} / \mathrm{cm}^{2}$. Such ferromagnetic coupling in the $\mathrm{Fe} / \mathrm{MgO} / \mathrm{Fe}$ system has already been reported, but with lower values than the present result [9,10]. In Ref. [9], the interface is suspected to be contaminated with carbon (no $\mathrm{MgO}$ buffer), which modifies both the chemical and topographical properties of the interface [20].

Anyway, such crossover from antiferromagnetic to ferromagnetic coupling is absolutely unexpected for an insulating barrier in the free-electron framework. It has been firstly attributed to an "orange peel" effect [9]. Thanks to the HRTEM observations which show very flat interfaces, the orange peel effect has to be ruled out in the present study. Later [10], the antiferromagnetic coupling was correlated to $\mathrm{O}$ vacancies, and the ferromagnetic coupling to the recovering of an ideal $\mathrm{MgO}$ barrier with increasing thickness. This interpretation is in contradiction with the present result, as the rough interface would present at the same time the characteristic of a "bad" interface with large $\mathrm{O}$ vacancies concentration (steeper antiferromagnetic coupling with respect to the flat interface) and of an "ideal" barrier (with recovering of a ferromagnetic coupling with increasing $\mathrm{MgO}$ thickness). As a matter of fact, the insertion of an atomic roughness exacerbates the exchange coupling mediated by $\mathrm{MgO}$, and reveals its oscillating behavior. It appears reminiscent of the well-known oscillations of the coupling across a metallic spacer. Concerning the IEC coupling across an insulating barrier, such oscillation has not yet been clearly theoretically predicted, either in the frame of the free-electron model or in band structure calculations of the tunneling probability. Nevertheless, in the latter case [41], the tunneling probability of electrons across a single-crystal barrier has an oscillating exponential decay. The oscillations arise from the quantum well resonances within the barrier related to the interference of the real part of the complex electronic wave vectors within the insulator. This effect has been clearly demonstrated experimentally in single-crystal magnetic tunnel junctions with the tunnel magnetoresistance oscillations as a function of $\mathrm{MgO}$ thickness [42] ranging between 1 and $3 \mathrm{~nm}$. Based on these theoretical and experimental results, one could extrapolate and predict similar oscillating exponential thickness decay for the field like equilibrium spin torque responsible for the experimentally measured IEC [43]. In terms of coupling, these oscillations could determine sign oscillation from antiferromagnetic to ferromagnetic.

On the other hand, from an experimental point of view, Matsumoto et al. have found that a 0.6-nm (3-ML) -thick $\mathrm{MgO}$ layer shows metallic transport properties [44] with a Giant Magneto-Resistance (GMR) effect, and that the tunnel barrier properties appear only for at least $4 \mathrm{ML}$. This was attributed to conductive paths in the $\mathrm{MgO}$ layer. Beyond this careful explanation, this metallic behavior could be correlated with the metalliclike temperature and spacer thickness dependences of the EXC coupling.

The observation of the successive metallic, semiconductive, and insulating behaviors with increasing $\mathrm{MgO}$ thickness suggests a more intrinsic property of this spacer, and reminds us of the progressive increase of the band gap as was already mentioned [45]. In order to support our assumption with respect to the gradual transition: from metal-semiconductorinsulator of the $\mathrm{MgO}$ barrier upon its thickness, we have performed $a b$ initio calculations using the WIEN2K code [46]. Our calculation model is based on a supercell slab composed of seven layers of Fe interfaced to seven layers of $\mathrm{MgO}$ [Fig. 8(a)]. This model allows us to investigate the layer-by-layer projected density of states. Therefore, from the spin-polarized layers projected DOS [Fig. 8(b)], one can clearly observe that a minimum of three atomic layers of insulator is required to get the opening of a gap. In the first atomic $\mathrm{MgO}$ plane (P1), close to the $\mathrm{Fe}$ interface, metal-induced gap states clearly exist. Therefore, a $\mathrm{Fe} / \mathrm{MgO} / \mathrm{Fe}$ structure with $2 \mathrm{ML}$ of $\mathrm{MgO}$ would behave as a metallic structure, a minimum five planes being necessary to get in the inner third plane insulatinglike behavior with a well-defined insulating band gap. In our experimental study, the $\mathrm{MgO}$ thickness regime is well below five atomic planes, and therefore no net insulating behavior is theoretically expected. The regime would rather correspond to a transition regime where the $\mathrm{MgO}$ would behave as a small-gap semiconductor with thermal activation properties of carrier transport into the conduction band.

In this framework, the steeper variation of the coupling with $t_{\mathrm{MgO}}$ for the atomic rough interface could be related to 



FIG. 8. (a) Supercell model used in ab initio calculation. (b) Layer projected density of states for up and down spins in the $\mathrm{Fe} / \mathrm{MgO} / \mathrm{Fe}$ supercell slab.

an enhancement of the metallic characteristic of the junction, resulting in an enhancement of the amplitude of the oscillations of a RKKY-type coupling. In a previous study performed with tunnel transport, a partial quenching of the Fe surface state with atomic islands has been observed [32]. This quenching could indeed increase the density of the conducting electrons, and then corroborate the stronger metallic behavior observed in the present study for the atomic rough interface.

Finally, we point out is that the graduate properties of the $\mathrm{MgO}$ layer on $\mathrm{Fe}(100)$ almost coincide with its specific epitaxy: the $\mathrm{MgO}$ is totally strained by the underlying buffer, as shown here by HRTEM and previous studies [32,45] until about 5 ML.

\section{CONCLUSION}

A systematic study of the interlayer exchange coupling in planar $\mathrm{Fe} / \mathrm{MgO} / \mathrm{Fe}$ (001) junctions has been performed as a function of temperature, $\mathrm{MgO}$ thickness, and interface morphology.

Two components of the coupling with opposite temperature dependence have been disentangled. The main contribution, so-called exchange coupling (EXC), which is present in the whole temperature and $\mathrm{MgO}$ thickness ranges, decreases with increasing temperature. This dependence is unexpected for an insulating barrier, and rather corresponds to a metallic behavior. The second contribution exhibits an opposite temperature dependence, well described by an Arrhenius law. This so-called temperature activated coupling is always antiferromagnetic, whatever the interface morphology.

Moreover, whereas the coupling strength depends not only on $\mathrm{MgO}$ thickness but also on the ferromagnetic electrodes and on the interface quality modulated by the insertion of Fe islands, we show that the parameters characterizing the temperature dependence of both contributions are almost independent of the stack, $\mathrm{MgO}$ thickness, and interface modulation. These parameters appear then as intrinsic characteristics of the coupling mechanism.
Interestingly, the insertion of an atomic roughness at the bottom $\mathrm{Fe} / \mathrm{MgO}$ interface reveals an oscillation of the direct exchange coupling with a crossover from antiferromagnetic to ferromagnetic around $4 \mathrm{MgO}$ ML. This behavior is reminiscent of the features of a RKKY interaction. As a matter of fact, the EXC coupling exhibits two signatures belonging to a metallic spacer: temperature decrease and oscillation with spacer thickness. This is reminiscent of the metallic transport properties and GMR effect already reported for $3 \mathrm{MgO}$ ML [44].

All these results suggest a new insight of the mechanism of the magnetic coupling in planar $\mathrm{Fe} / \mathrm{MgO}$ junctions: from metallic to semiconducting behavior as a result of a progressive opening of the gap with increasing $\mathrm{MgO}$ thickness. This insight is supported by ab initio calculations, performed with a $\mathrm{Fe} / \mathrm{MgO} / \mathrm{Fe}$ supercell, which show that a minimum spacer thickness of five planes is necessary to get huge insulating properties in the third inner $\mathrm{MgO}$ plane.

\section{ACKNOWLEDGMENTS}

C.B. is thankful to Jérôme Eugène for his help and collaboration. This work was supported partly by the french PIA project "Lorraine Université d'Excellence," reference ANR-15-IDEX-04-LUE.

\section{APPENDIX A: ANALYTICAL MODEL FOR ANTIFERROMAGNETIC COUPLED BILAYER}

We consider two magnetic layers with a fourfold anisotropy, $K_{1}, K_{2}$ being the anisotropies of layers 1 and 2 of thickness $t_{1}$ and $t_{2}$, and magnetizations $\mathbf{M}_{\mathbf{1}}$ and $\mathbf{M}_{\mathbf{2}}$. The antiferromagnetic bilinear coupling is $J<0$, and biquadratic coupling $J_{b}<$ $0, \theta_{1,2}$ represent the angles between the magnetizations $\mathbf{M}_{\mathbf{1}, 2}$ and the field $\mathbf{H}, \varphi$ the angle between one of the easy axes and the field. Figure 9 presents schematically the macrospin Stoner-Wohlfarth model used in the modeling.

We follow below the treatment of Tiusan et al. [47] with the addition of a biquadratic coupling term.

In the frame of the Stoner-Wohlfarth model, with an applied field along an easy axis $(\varphi=0)$, the total energy per surface unit is given by

$$
\begin{aligned}
E_{\text {totale }}= & -M_{\mathrm{Fe}} H\left(t_{1} \cos \theta_{1}+t_{2} \cos \theta_{2}\right)-J \cos \left(\theta_{1}-\theta_{2}\right) \\
& -J_{b} \cos ^{2}\left(\theta_{1}-\theta_{2}\right)+\frac{K_{1} t_{1}}{4} \sin ^{2} 2 \theta_{1}+\frac{K_{2} t_{2}}{4} \sin ^{2} 2 \theta_{2} .
\end{aligned}
$$

$M_{\mathrm{Fe}}$ is the magnetization of both layers.
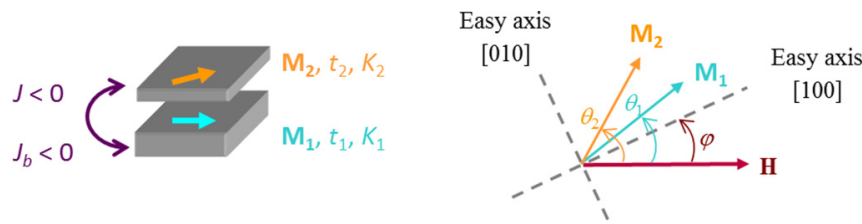

FIG. 9. Sketch of the bilayer illustrating the angles $\theta_{1}, \theta_{2}$, and $\varphi ; K_{i}$ : fourfold anisotropy constant of layer $i ; M_{i}$ : magnetization of layer $i, t_{i}$ : thickness of layer $i ; J$ : bilinear coupling; $J_{b}$ : biquadratic coupling. 
The minimization of the energy with respect to the angles $\theta_{1}$ and $\theta_{2}$ provides

$$
\begin{aligned}
\sin \left(\theta_{2}-\theta_{1}\right)\left[J+2 J_{b} \cos \left(\theta_{2}-\theta_{1}\right)\right] & \\
= & H M_{\mathrm{Fe}} t_{1} \sin \theta_{1}+K_{1} t_{1} \sin 2 \theta_{1} \cos 2 \theta_{1} . \\
& -\sin \left(\theta_{2}-\theta_{1}\right)\left[J+2 J_{b} \cos \left(\theta_{2}-\theta_{1}\right)\right] \\
= & H M_{\mathrm{Fe}} t_{2} \sin \theta_{2}+K_{2} t_{2} \sin 2 \theta_{2} \cos 2 \theta_{2},
\end{aligned}
$$

The saturation and plateau fields, $H_{S}$ and $H_{P}$, correspond to the limits $\left\{\theta_{1} \rightarrow 0^{-} ; \theta_{2} \rightarrow 0^{+}\right\}$and $\left\{\theta_{1} \rightarrow 0^{-} ; \theta_{2} \rightarrow \pi^{-}\right\}$, respectively. The same methodology as used by Tiusan et al. [47] gives

$$
\begin{aligned}
-1 & =\frac{J+2 J_{b}}{t_{1}} \frac{1}{M_{\mathrm{Fe}} H_{S}+2 K_{1}}+\frac{J+2 J_{b}}{t_{2}} \frac{1}{M_{\mathrm{Fe}} H_{S}+2 K_{2}}, \\
1 & =\frac{J-2 J_{b}}{t_{1}} \frac{1}{M_{\mathrm{Fe}} H_{P}+2 K_{1}}-\frac{J-2 J_{b}}{t_{2}} \frac{1}{M_{\mathrm{Fe}} H_{P}-2 K_{2}} .
\end{aligned}
$$

These expressions can be written as

$$
\begin{aligned}
-1 & =\frac{J+2 J_{b}}{2 K_{1} t_{1}} \frac{1}{\frac{M_{\mathrm{Fe}} H_{S}}{2 K_{1}}+1}+\frac{J+2 J_{b}}{2 K_{2} t_{2}} \frac{1}{\frac{M_{\mathrm{Fe}} H_{S}}{2 K_{2}}+1}, \\
1 & =\frac{J-2 J_{b}}{2 K_{1} t_{1}} \frac{1}{\frac{M_{\mathrm{Fe}} H_{P}}{2 K_{1}}+1}-\frac{J-2 J_{b}}{2 K_{2} t_{2}} \frac{1}{\frac{M_{\mathrm{Fe}} H_{P}}{2 K_{2}}-1} .
\end{aligned}
$$

With the introduction of the anisotropy fields of layers 1 and 2 as

$$
\begin{aligned}
H_{a 1(a 2)} & =2 K_{1(2)} / M_{\mathrm{Fe}}, \text { one gets } \\
-1 & =\frac{J+2 J_{b}}{2}\left(\frac{1}{K_{1} t_{1}} \frac{1}{\frac{H_{S}}{H_{a 1}}+1}+\frac{1}{K_{2} t_{2}} \frac{1}{\frac{H_{S}}{H_{a 2}}+1}\right), \\
1 & =\frac{J-2 J_{b}}{2}\left(\frac{1}{K_{1} t_{1}} \frac{1}{\frac{H_{P}}{H_{a 1}}+1}-\frac{1}{K_{2} t_{2}} \frac{1}{\frac{H_{P}}{H_{a 2}}-1}\right) .
\end{aligned}
$$

In the case of a bilayer with $t_{1} \gg t_{2}$ as a thick/thin stack, or $K_{1} \gg K_{2}$ as a soft/hard stack, some terms are negligible and one obtains

$$
\begin{aligned}
-1 & \approx \frac{J+2 J_{b}}{2}\left(\frac{1}{K_{2} t_{2}} \frac{1}{\frac{H_{S}}{H_{a 2}}+1}\right), \\
1 & \approx \frac{J-2 J_{b}}{2}\left(-\frac{1}{K_{2} t_{2}} \frac{1}{\frac{H_{P}}{H_{a 2}}-1}\right) .
\end{aligned}
$$

The plateau and saturation fields can then be expressed as

$$
\begin{aligned}
& H_{S}+\frac{2 K_{2}}{M_{\mathrm{Fe}}}=-\frac{1}{t_{2} M_{\mathrm{Fe}}}\left(J+2 J_{b}\right), \\
& H_{P}-\frac{2 K_{2}}{M_{\mathrm{Fe}}}=-\frac{1}{t_{2} M_{\mathrm{Fe}}}\left(J-2 J_{b}\right) .
\end{aligned}
$$

In the case of the absence of coupling $J=J_{b}=0$, the plateau and saturation fields correspond to the coercive fields of the single thin layer in the frame of the Stoner-Wohlfarth model with coherent rotation of the magnetic moment of the layers:

$$
H_{S}=-\frac{2 K_{2}}{M_{\mathrm{Fe}}}, \quad H_{P}=+\frac{2 K_{2}}{M_{\mathrm{Fe}}} .
$$

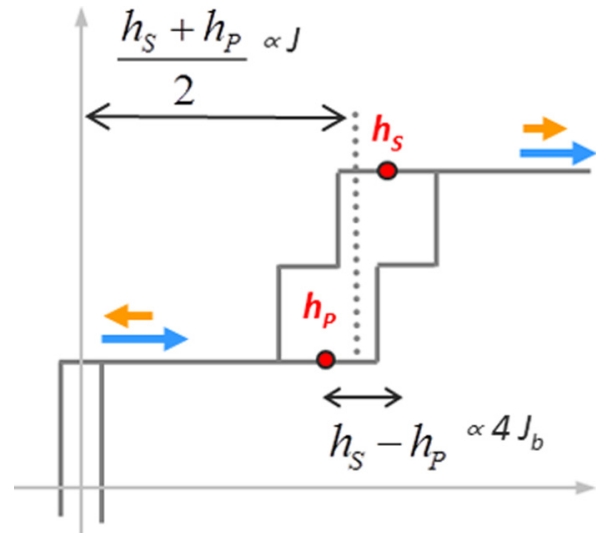

FIG. 10. Sketch of an experimental minor loop of the thin layer.

Nevertheless, experimentally, with an applied field along the easy axis, the reversing of the layer moment occurs with nucleation and propagation of domain walls. The opening of the minor loop is then much lower than $\frac{4 K_{2}}{M_{\mathrm{Fe}}}$, as illustrated in Fig. 10 .

The measured saturation and plateau fields, $h_{s}$ and $h_{p}$, are then related to the coupling constants by

$$
h_{S}=-\frac{1}{t_{2} M_{\mathrm{Fe}}}\left(J+2 J_{b}\right), \quad h_{P}=-\frac{1}{t_{2} M_{\mathrm{Fe}}}\left(J-2 J_{b}\right) .
$$

From these relations, one can deduce

$$
J=-M_{\mathrm{Fe}} t_{2} \frac{h_{S}+h_{P}}{2}, \quad J_{b}=-\frac{M_{\mathrm{Fe}} t_{2}}{2} \frac{h_{S}-h_{P}}{2} .
$$

\section{APPENDIX B: BIQUADRATIC COUPLING}

This Appendix gathers both the temperature and artificial roughness dependence of the biquadratic coupling.

The temperature dependence of the effective biquadratic coupling measured with the thin/thick stack and flat interface is plotted in Fig. 11. The corresponding bilinear coupling has been plotted in Fig. 3 .

First of all, one can point out that this biquadratic coupling is about one order of magnitude lower than the bilinear coupling. For this reason, it has not been included in the main discussion of the paper. For a 3-ML MgO spacer, it increases almost linearly from about $40 \%$ with decreasing temperature, whereas

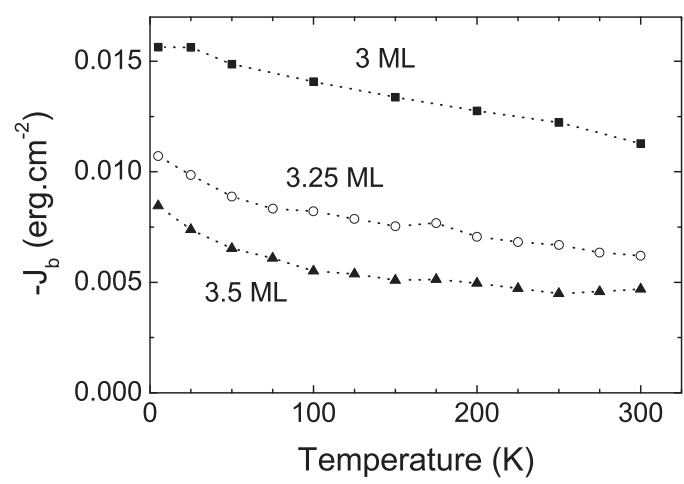

FIG. 11. Temperature dependence of the biquadratic coupling of three $\mathrm{Fe}(50 \mathrm{~nm}) / \mathrm{MgO}(x \mathrm{~nm}) / \mathrm{Fe}(5 \mathrm{~nm})$ junctions with $x=3.0 \mathrm{ML}$; 3.25 ML; 3.5 ML. 


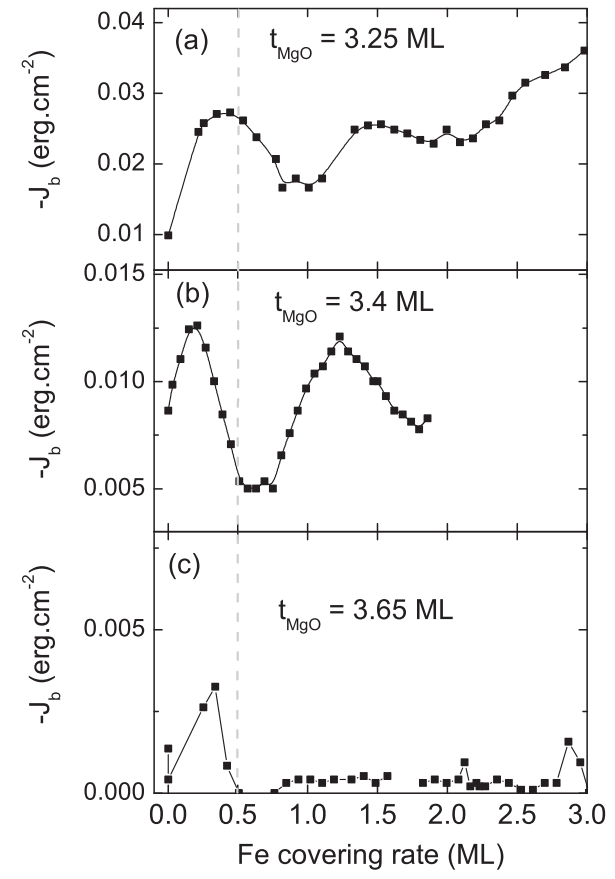

FIG. 12. Biquadratic coupling constant measured at room temperature by Kerr magnetometry as a function of the Fe covering rate of the Fe buffer for three MgO spacer thicknesses: (a) 3.25 ML; (b) 3.4 ML; (c) $3.65 \mathrm{ML}$. The stack of the sample is Fe $(50 \mathrm{~nm}) / \mathrm{MgO}$ $(t) / \mathrm{Fe}(5 \mathrm{~nm})$.

a stronger increase is observed below $100 \mathrm{~K}$ in the case of 3.25 and $3.5 \mathrm{MgO} \mathrm{ML}$. This feature coincides with the occurrence of the Arrhenius term in the bilinear coupling. As a consequence, different mechanisms should contribute to the biquadratic coupling. It could arise from spatial fluctuations of the bilinear coupling due to thickness fluctuations of the spacer [48]. It could be also interpreted in the frame of the loose spins model of Slonczewski [49], which was also used in [12]. The coupling is then supported by magnetic impurity at the interface or inside the barrier.

Figure 12 gathers the $\mathrm{Fe}$ coverage dependence of the biquadratic coupling measured at $300 \mathrm{~K}$. The corresponding bilinear coupling is plotted in Fig. 5. Here again, for a Fe coverage lower than $1 \mathrm{ML}$, the biquadratic coupling is one order of magnitude lower than the bilinear one. Moreover, in this Fe covering range $(<1 \mathrm{ML})$, an oscillation is present for 3.25 and $3.4 \mathrm{MgO} \mathrm{ML}$, which is perfectly reminiscent of the oscillation of the bilinear coupling. It can therefore be attributed to the fourfold anisotropy [33] or a distribution effect of the bilinear component. On the contrary, for larger $\mathrm{Fe}$ coverage, close to $3 \mathrm{Fe} \mathrm{ML}$, it increases whereas the oscillations of the bilinear coupling are damped (Fig. 5). This could be readily attributed to an increasing fluctuation mechanism with artificial roughness or Fe coverage [48].
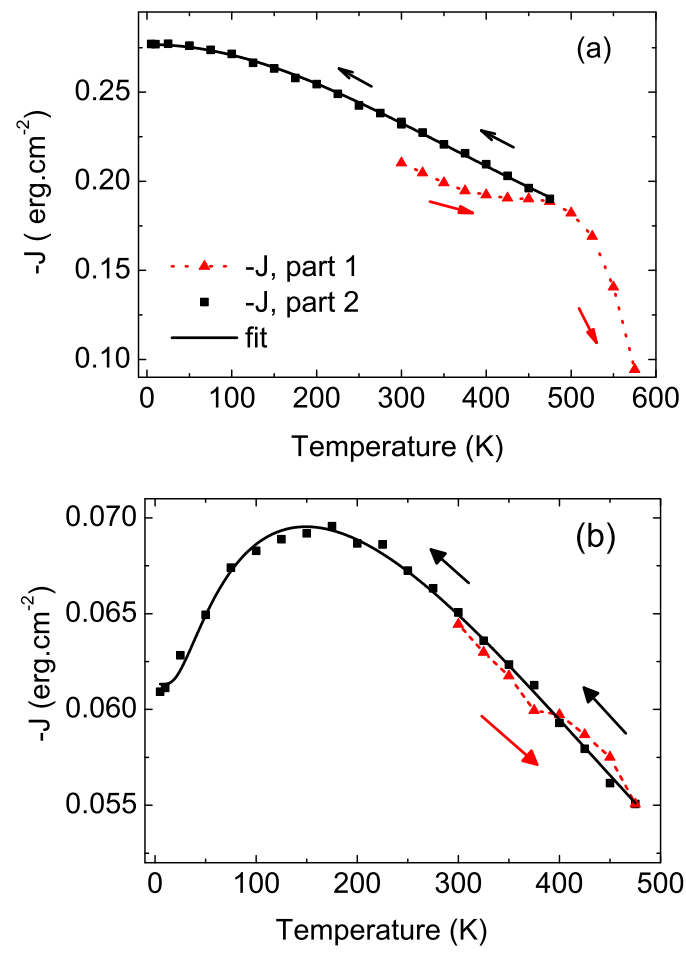

FIG. 13. Extended temperature dependence of $-J$ of two $\mathrm{Fe}$ $(50 \mathrm{~nm}) / \mathrm{MgO}(x \mathrm{~nm}) / \mathrm{Fe}(5 \mathrm{~nm})$ junctions with (a) $x=3.0 \mathrm{ML}$; (b) $3.5 \mathrm{ML}$. The straight lines are fits with the model described in the text. The red points correspond to measurements performed with increasing temperature, the black one, with decreasing temperature.

\section{APPENDIX C: HIGH-TEMPERATURE MEASUREMENTS}

In order to extend the study of the temperature dependence beyond the $0-300 \mathrm{~K}$ range, the coupling has been investigated in the SQUID-VSM magnetometer using an oven stick.

Figures 13(a) and 13(b) show the temperature dependence of the coupling strength of a thick/thin stack with a 3- (3.5-) ML $\mathrm{MgO}$ spacer. First of all, in order to point out annealing effects with increasing temperature, measurements have been performed with part 1 of the sample until its complete breaking (red points). The quality of the barrier is preserved and even enhanced until $475 \mathrm{~K}$. The temperature dependence of the coupling intensity has then been studied with part 2 of the sample in the range 5-475 K (black points) with decreasing temperature. The straight line corresponds to the fit with formula (2) in Fig. 13(a) and with formula (3) in Fig. 13(b), corresponding to a thicker $\mathrm{MgO}$ spacer. The model of Bruno predicts an increase of the coupling with temperature in the case of an insulating spacer [2]. Such behavior has not been observed; the fitting with the metal-spacer model and an Arrhenius law is satisfactory in the whole temperature range.
[1] P. Bruno, J. Magn. Magn. Mater. 121, 248 (1993).

[2] P. Bruno, Phys. Rev. B 52, 411 (1995).
[3] M. Landolt and B. Briner, Appl. Phys. A: Mater. Sci. Process. 60, 403 (1995). 
[4] M. Hunziker and M. Landolt, Phys. Rev. B 64, 134421 (2001).

[5] R. R. Gareev, L. L. Pohlmann, S. Stein, D. E. Bürgler, P. A. Grünberg, and M. Siegel, J. Appl. Phys. 93, 8038 (2003).

[6] A. Dinia, P. Carrof, G. Schmerber, and C. Ulhacq, Appl. Phys. Lett. 83, 2202 (2003).

[7] D. E. Bürgler, R. R. Gareev, L. L. Pohlmann, H. Braak, M. Buchmeier, M. Luysberg, R. Schreiber, and P. A. Grünberg, Magnetic Nanostructures, Springer Series in Materials Science, Vol. 94 (Springer, Berlin, Heidelberg, 2007), p. 133.

[8] S. J. Carreira, L. A. Félix, M. Sirena, G. Alejandro, and L. B. Steren, Appl. Phys. Lett. 109, 062402 (2016).

[9] J. Faure-Vincent, C. Tiusan, C. Bellouard, E. Popova, M. Hehn, F. Montaigne, and A. Schuhl, Phys. Rev. Lett. 89, 107206 (2002).

[10] T. Katayama, S. Yuasa, J. Velev, M. Ye. Zhuralev, S. S. Jaswal, and E. Y. Tsymbal, Appl. Phys. Lett. 89, 112503 (2006).

[11] H.-C. Wu, S. K. Arora, O. N. Mryasov, and I. V. Shvets, Appl. Phys. Lett. 92, 182502 (2008).

[12] Y. F. Chiang, J. J. I. Wong, X. Tan, Y. Li, K. Pi, W. H. Wang, H. W. K. Tom, and R. K. Kawakami, Phys. Rev. B 79, 184410 (2009).

[13] W. Skowronski, T. Stobiecki, J. Wrona, K. Rott, A. Thomas, G. Reiss, and S. Van Dijken, J. Appl. Phys. 107, 093917 (2010).

[14] L. E. Nistor, B. Rodmacq, S. Auffret, A. Schuhl, M. Chshiev, and B. Dieny, Phys. Rev. B 81, 220407 (2010).

[15] L. Li, F. Zhang, N. Wang, Y. F. Lv, X. Y. Han, and J. J. Zhang, J. Appl. Phys. 108, 073908 (2010).

[16] J. J. I. Wong, L. Ramirez, A. G. Swartz, A. Hoff, W. Han, Y. Li, and R. K. Kawakami, Phys. Rev. B 81, 094406 (2010).

[17] S. Serrano-Guisan, W. Skowronski, J. Wrona, N. Liebing, M. Czapkiewicz, T. Stobiecki, G. Reiss, and H. W. Schumacher, J. Appl. Phys. 110, 023906 (2011).

[18] Y. Fan, K. J. Smith, G. Lüpke, A. T. Hanbicki, R. Goswami, C. H. Li, H. B. Zhao, and B. T. Jonker, Nat. Nanotechnol. 8, 438 (2013).

[19] Y.-C. Weng, C.-W. Cheng, and G. Chern, IEEE Trans. Magn. 49, 4425 (2013).

[20] A. Kozio-Rachwa, T. Izak, M. Izak, K. Matlak, E. Myczak, N. Spiridis, and J. Korecki, J. Appl. Phys. 115, 104301 (2014).

[21] L. Li, D. Han, W. Lei, Z. Liu, F. Zhang, X. Mao, P. Wang, and H. Hou, J. Appl. Phys. 116, 123904 (2014).

[22] R. R. Gareev, V. Zbarsky, J. Landers, I. Soldatov, R. Schäfer, M. Münzenberg, H. Wende, and P. Grünberg, Appl. Phys. Lett. 106, 132408 (2015).

[23] R. Moubah, F. Magnus, T. Warnatz, G. K. Palsson, V. Kapaklis, V. Ukleev, A. Devishvili, J. Palisaitis, P. O. A. Persson, and B. Hjorvarsson, Phys. Rev. Appl. 5, 044011 (2016).

[24] A. A. Baker, A. I. Figueroa, D. Pingstone, V. K. Lazarov, G. van der Laan, and T. Hesjedal, Sci. Rep. 6, 35582 (2016).

[25] H. Yanagihara, Y. Toyoda, and E. Kita, J. Phys. D: Appl. Phys. 44, 064011 (2011).
[26] J. C. Slonczewski, Phys. Rev. B 39, 6995 (1989).

[27] P. Bruno, Phys. Rev. B 49, 13231 (1994).

[28] M. Y. Zhuravlev, E. Y. Tsymbal, and A. V. Vedyayev, Phys. Rev. Lett. 94, 026806 (2005)

[29] M. Y. Zhuravlev, J. P. Velev, A. Vedyayev, and E. Y. Tsymbal, J. Magn. Magn. Mater. 300, e277 (2006).

[30] H. X. Yang, M. Chshiev, A. Kalitsov, A. Schuhl, and W. H. Butler, Appl. Phys. Lett. 96, 262509 (2010).

[31] M. Sicot, S. Andrieu, C. Tiusan, F. Bertran, and F. Montaigne, J. Appl. Phys. 99, 08D301 (2006).

[32] A. Duluard, C. Bellouard, Y. Lu, M. Hehn, D. Lacour, F. Montaigne, G. Lengaigne, S. Andrieu, F. Bonell, and C. Tiusan, Phys. Rev. B 91, 174403 (2015).

[33] C. Bellouard, J. Faure-Vincent, C. Tiusan, F. Montaigne, M. Hehn, V. Leiner, H. Fritzsche, and M. Gierlings, Phys. Rev. B 78, 134429 (2008).

[34] B. Briner and M. Landolt, Phys. Rev. Lett. 73, 340 (1994).

[35] P. Walser, M. Schleberger, P. Fuchs, and M. Landolt, Phys. Rev. Lett. 80, 2217 (1998).

[36] Z. Zhang, L. Zhou, P. E. Wigen, and K. Ounadjela, Phys. Rev. Lett. 73, 336 (1994).

[37] N. S. Almeida, D. L. Mills, and M. Teitelman, Phys. Rev. Lett. 75, 733 (1995).

[38] N. Persat and A. Dinia, Phys. Rev. B 56, 2676 (1997).

[39] C.-L. Lee, J. A. Bain, S. Chu, and M. E. McHenry, J. Appl. Phys. 91, 7113 (2002).

[40] S.-H. Yang, B. Balke, C. Papp, S. Doring, U. Berges, L. Plucinski, C. Westphal, C. M. Schneider, S. S. P. Parkin, and C. S. Fadley, Phys. Rev. B 84, 184410 (2011).

[41] W. H. Butler, X.-G. Zhang, T. C. Schulthess, and J. M. MacLaren, Phys. Rev. B 63, 054416 (2001).

[42] S. Yuasa, T. Nagahama, A. Fukushima, Y. Suzuki, and K. Ando, Nat. Mater. 3, 868 (2004).

[43] M. Chshiev, A. Manchon, A. Kalitsov, N. Ryzhanova, A. Vedyayev, N. Strelkov, W. H. Butler, and B. Dieny, Phys. Rev. B 92, 104422 (2015).

[44] R. Matsumoto, A. Fukushima, K. Yakushiji, S. Yakata, T. Nagahama, H. Kubota, T. Katayama, Y. Suzuki, K. Ando, S. Yuasa, B. Georges, V. Cros, J. Grollier, and A. Fert, Phys. Rev. B 80, 174405 (2009).

[45] M. Klaua, D. Ullmann, J. Barthel, W. Wulfhekel, J. Kirschner, R. Urban, T. L. Monchesky, A. Enders, J. F. Cochran, and B. Heinrich, Phys. Rev. B 64, 134411 (2001).

[46] P. Blaha, K. Schwarz, G. K. H. Madsen, D. Kvasnicka, and J. Luitz, WIEN2K, An Augmented Plane Wave Local Orbitals Program for Calculating Crystal Properties (Technical University of Wien, Austria, 2001).

[47] C. Tiusan, F. Greullet, M. Hehn, F. Montaigne, S. Andrieu, and A. Schuhl, J. Phys.: Condens. Matter 19, 165201 (2007).

[48] J. C. Slonczewski, Phys. Rev. Lett. 67, 3172 (1991).

[49] J. C. Slonczewski, J. Appl. Phys. 73, 5957 (1993). 